\title{
Metabolic engineering and flux analysis of Corynebacterium glutamicum for L-serine production
}

\author{
LAI ShuJuan ${ }^{1,2}$, ZHANG Yun ${ }^{1}$, LIU ShuWen ${ }^{1,2}$, LIANG Yong ${ }^{1}$, SHANG XiuLing ${ }^{1,2}$, \\ CHAI Xin ${ }^{1,2} \&$ WEN Ting $\mathrm{Yi}^{1^{*}}$ \\ ${ }^{1}$ Department of Industrial Microbiology and Biotechnology, Institute of Microbiology, Chinese Academy of Sciences, Beijing 100101, China; \\ ${ }^{2}$ Graduate University of Chinese Academy of Sciences, Beijing 100049, China
}

\begin{abstract}
L-Serine plays a critical role as a building block for cell growth, and thus it is difficult to achieve the direct fermentation of L-serine from glucose. In this study, Corynebacterium glutamicum ATCC 13032 was engineered de novo by blocking and attenuating the conversion of L-serine to pyruvate and glycine, releasing the feedback inhibition by L-serine to 3-phosphoglycerate dehydrogenase (PGDH), in combination with the co-expression of 3-phosphoglycerate kinase (PGK) and feedback-resistant PGDH $\left(\mathrm{PGDH}^{\mathrm{r}}\right)$. The resulting strain, SER-8, exhibited a lower specific growth rate and significant differences in L-serine levels from Phase I to Phase V as determined for fed-batch fermentation. The intracellular L-serine pool reached $(14.22 \pm 1.41) \mu \mathrm{mol} \mathrm{g}_{\mathrm{CDM}}{ }^{-1}$, which was higher than glycine pool, contrary to fermentation with the wild-type strain. Furthermore, metabolic flux analysis demonstrated that the over-expression of PGK directed the flux of the pentose phosphate pathway (PPP) towards the glycolysis pathway (EMP), and the expression of $\mathrm{PGDH}^{\mathrm{r}}$ improved the L-serine biosynthesis pathway. In addition, the flux from L-serine to glycine dropped by $24 \%$, indicating that the deletion of the activator GlyR resulted in down-regulation of serine hydroxymethyltransferase (SHMT) expression. Taken together, our findings imply that L-serine pool management is fundamental for sustaining the viability of $C$. glutamicum, and improvement of $\mathrm{C}_{1}$ units generation by introducing the glycine cleavage system $(\mathrm{GCV})$ to degrade the excessive glycine is a promising target for L-serine production in C. glutamicum.
\end{abstract}

Corynebacterium glutamicum, L-serine, intracellular metabolites, metabolic engineering, elementary mode analysis

Citation: Lai S J, Zhang Y, Liu S W, et al. Metabolic engineering and flux analysis of Corynebacterium glutamicum for L-serine production. Sci China Life Sci, 2012, 55: 283-290, doi: 10.1007/s11427-012-4304-0

The amino acid L-serine is one of the major components for nutritional growth and development of the central nervous system of mammals [1,2], but is also a predominant source of one-carbon $\left(\mathrm{C}_{1}\right)$ units in de novo biosynthesis of purines, amino acids and thymidylate [3-5] as well as an intermediate for phospholipid biosynthesis [6] in a variety of organisms. Given its critical role as a building block for cell growth, the bacterium Corynebacterium glutamicum, for example, has as much as $7.5 \%$ of its total glucose-originat-

*Corresponding author (email: wenty@im.ac.cn) ing carbon flux distributed to L-serine biosynthesis [7]. In C. glutamicum, L-serine biosynthesis is initiated from the glycolytic intermediate 3-phosphoglycerare and carried through three sequential reactions catalyzed by 3-phosphoglycerate dehydrogenase (PGDH), 3-phosphoserine aminotransferase (PSAT) and 3-phosphoserine phosphatase (PSP), respectively. PGDH is the gene product of serA and catalyzes the oxidation of 3-phosphoglycerare to generate 3-phosphohydroxypyruvate. PSAT is encoded by serC and catalyzes the transamination of 3-phosphohydroxypyruvate to yield 3-phosphoserine. 3-Phophoserine is then dephosp- 
horylated to yield L-serine catalyzed by PSP, a gene product of $\operatorname{ser} B$ (Figure 1). Among the three enzymes, PDGH is found to be sensitive to feedback inhibition by L-serine and only remains $60 \%$ activity in the presence of $10 \mathrm{mmol} \mathrm{L}^{-1}$ L-serine [8]. It has also been identified that the carboxy-terminal 197 amino acids of PGDH are responsible for the feedback inhibition by L-serine and its deletion has only a minimal effect on the enzyme activity [8].

Catabolism of L-serine in C. glutamicum proceeds via pyruvate in the presence of glucose. This conversion reaction is catalyzed by L-serine dehydratase (L-SerDH) encoded by the gene $s d a A$ [9]. L-Serine is also used to provide building blocks for amino acid biosynthesis including glycine, cysteine and tryptophan [9-11]. Isotope tracer analysis demonstrated that the carbon skeleton of ${ }^{13} \mathrm{C}$-labeled $\mathrm{L}$-serine is mainly converted to glycine [9]. This reaction is catalyzed by the gene product of $g l y A$, a serine hydroxymethyltransferase (SHMT) [12]. SHMT catalyzes the reversible, simultaneous conversion of L-serine to glycine and tetrahydrofolate to 5,10-methylenetetrahydrofolate. Since this reaction provides the majority of the $\mathrm{C}_{1}$ units needed for cell growth, disruption of the glyA gene is lethal to host cells [12]. A recent study identified that, in the stationary growth phase, glyA gene expression is tightly controlled by an acti-

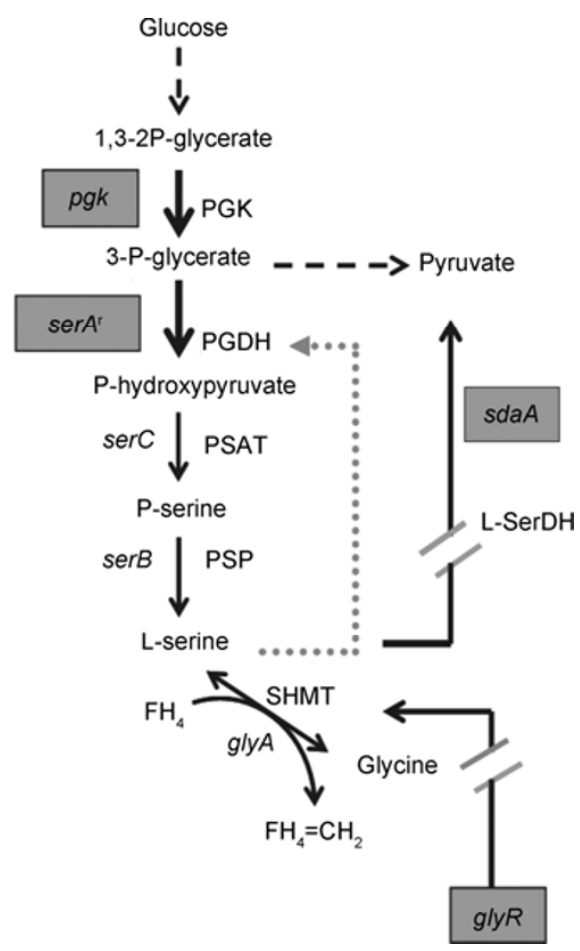

Figure 1 Strategy to engineer $C$. glutamicum for L-serine production. Genetic manipulation includes the inactivation of the catabolic genes $s d a A$ and $g l y R$ (double short lines), the release of the feedback inhibition of 3-phosphoglycerate dehydrogenase (PGDH) by L-serine (dotted long arrow) and the over-expression of the biosynthesis genes $p g k$ and feedback-resistant $\operatorname{serA}^{\mathrm{r}}$ (boldfaced arrows). 1,3-2P-glycerate: 1,3-diphosphoglycerate; 3P-glycerate: 3-phosphoglycerate; P-hydroxypyruvate: phosphohydroxypyruvate; P-serine: phosphoserine; $\mathrm{FH}_{4}$ : tetrahydrofolate and $\mathrm{FH}_{4}=\mathrm{CH}_{2}$ : 5,10-methylenetetrahydrofolate. vator GlyR at the transcriptional level [13]. This finding provides a possible regulatory tool to control the flux from L-serine to glycine by manipulating GlyR expression.

At present, the industrial production of L-serine mainly depends on the enzymatic or cellular conversion of the substrate glycine and a $\mathrm{C}_{1}$ compound in the presence of an enzyme system composed of SHMT [14]. Many methanol-utilizing bacteria, such as Hyphomicrobium methylovorum, have been applied to produce L-serine by using glycine and methanol [15]. These systems either use expensive substrates or exhibit low productivity that extremely restricts their application for L-serine production. In addition, using these industrial strains, large-scale fermentation for L-serine production is not achieved and unlike the production of other amino acids, the conversion of cheap sugar to L-serine is very difficult.

C. glutamicum is amenable to genetic modification and shows robust fermentation characteristics using glucose as a carbon source, hence making it a good candidate to produce amino acids $[16,17]$. Subjected to systems metabolic engineering in recent years, $C$. glutamicum has been successfully applied to produce amino acids including L-lysine, L-valine and L-methionine [17-19]. However, the L-serine production from this bacterium is not yet suitable for industrial application [20], mainly due to the fact that the metabolic regulatory systems for intracellular L-serine accumulation remain largely unknown. In this study, we constructed an engineered strain SER-8, quantified its extra- and intra-cellular products and analyzed its carbon flux redistributions by constructing elementary modes to obtain functional insights into the performance of metabolic networks.

\section{Materials and methods}

\subsection{Bacterial strains, plasmids, and cell growth}

All bacterial strains and plasmids used in this study are listed in Table 1. Luria-Bertani medium was used for $E$. coli at $37^{\circ} \mathrm{C}$. Brain heart infusion medium was used as a complex medium for C. glutamicum at $30^{\circ} \mathrm{C}$. As a minimal medium, CGX medium was used with $40 \mathrm{~g} \mathrm{~L}^{-1}$ glucose [24]. If necessary, antibiotics were added at the following concentrations: $100 \mu \mathrm{g} \mathrm{mL}^{-1}$ ampicillin, $50 \mu \mathrm{g} \mathrm{mL}^{-1}$ kanamycin or $20 \mu \mathrm{g} \mathrm{mL}^{-1}$ chloramphenicol for $E$. coli and $25 \mu \mathrm{g} \mathrm{mL} \mathrm{m}^{-1}$ kanamycin or $10 \mu \mathrm{g} \mathrm{mL}^{-1}$ chloramphenicol for C. glutamicum. The strains harboring the plasmid with the $\mathrm{P}_{t a c}$ promoter were cultivated with the addition of $1 \mathrm{mmol} \mathrm{L}^{-1}$ isopropyl- $\beta$-D-thiogalactoside (IPTG).

\subsection{Construction of plasmids and strains}

All genetic modifications were introduced into the $C$. glutamicum genome using the homologous $s a c B$ recombination system [25,26]. Gene disruption was performed as described previously [27]. Correct integration and in-frame 
Table 1 Strains and plasmids used in this study

\begin{tabular}{|c|c|c|}
\hline Strains and plasmids & Relevant characteristics & Reference or source \\
\hline \multicolumn{3}{|l|}{ E. coli } \\
\hline $\mathrm{DH} 5 \alpha$ & $\begin{array}{c}\text { sup } \mathrm{E} 44, \varphi 80 \text { lacZ } \Delta \mathrm{M} 15, \text { hsdR } 17, \text { recA } 1, \text { endA1, } \\
\text { gyrA96,thi-1,relA } 1\end{array}$ & [21] \\
\hline \multicolumn{3}{|l|}{ C. glutamicum } \\
\hline WT & Wild-type, ATCC 13032 & $\mathrm{ATCC}^{\mathrm{a})}$ \\
\hline SER-0 & WT/pXMJ19 & This work \\
\hline SER-1 & $\mathrm{WT}-\Delta s d a A$ & This work \\
\hline SER-2 & WT- $\Delta s d a A \Delta g l y R$ & This work \\
\hline SER-3 & WT- $\Delta s d a A \Delta g l y R \operatorname{ser}^{\mathrm{r}}$ & This work \\
\hline SER-4 & SER-3/pXMJ19 & This work \\
\hline SER-5 & SER-3/pWYE1115 & This work \\
\hline SER-6 & SER-3/pWYE1116 & This work \\
\hline SER-7 & SER-3/pWYE1117 & This work \\
\hline SER-8 & SER-3/pWYE1118 & This work \\
\hline \multicolumn{3}{|l|}{ Plasmids } \\
\hline pK18mobsacB & $\mathrm{Km}^{\mathrm{r} b}$, containing $l a c I^{\mathrm{q}}$ & {$[22]$} \\
\hline pXMJ19 & Shuttle vector of E. coli and C. glutamicum. $\mathrm{Cm}^{\mathrm{r} b)}$ & [23] \\
\hline pWYE260 & $\mathrm{pK} 18 \mathrm{mobsacB}-\Delta s d a A$ & This work \\
\hline pWYE297 & pK18mobsacB- $\Delta$ glyR & This work \\
\hline pWYE1101 & pK18mobsacB-serA ${ }^{\mathrm{r}}$ & This work \\
\hline pWYE1115 & pXMJ19-pgk & This work \\
\hline pWYE1116 & pXMJ19-serA ${ }^{\mathrm{r}}$ & This work \\
\hline pWYE1117 & pXMJ19-pgk-serA ${ }^{\mathrm{r}}$ & This work \\
\hline pWYE1118 & pXMJ19-serA ${ }^{\mathrm{r}}-p g k$ & This work \\
\hline
\end{tabular}

a) ATCC, American Type Culture Collection. b) $\mathrm{Km}^{\mathrm{r}}$, kanamycin resistance; $\mathrm{Cm}^{\mathrm{r}}$, chloramphenicol resistance.

gene deletion were verified by PCR and sequencing. The genes were amplified with the primers listed in Appendix Table 1 in the electronic version. The PCR product was sub-cloned into the pMD19-T vector (Takara, Japan). The resultant plasmid was digested with $X b a \mathrm{I} / K p n \mathrm{I}$, and the $p g k$ or $\operatorname{ser} A^{\mathrm{r}}$-containing insert was ligated into $\mathrm{Xba} \mathrm{I} / K p n$ I-treated pXMJ19 to construct pWYE1115 (pXMJ19-pgk) and pWYE1116 (pXMJ19-serA ${ }^{\mathrm{r}}$ ), respectively. Considering the polar effect, two other plasmids harboring $p g k$ and $s e r A^{\mathrm{r}}$ in different tandem orders were constructed as follows: the pMD19-T-serA ${ }^{\mathrm{r}}$-R was digested with $K p n$ I/EcoR I and ligated into pWYE1115 to construct pWYE1117 (pXMJ19$p g k-s e r A^{\mathrm{r}}$ ). The pMD19-T-pgk-R was digested with Kpn I/ $E c o$ R I and ligated into pWYE1116 to construct pWYE1118 (pXMJ19-serA $\left.{ }^{\mathrm{r}}-p g k\right)$.

\subsection{Fermentation in shake flasks and a bioreactor}

In the shake-flask growth experiment, $C$. glutamicum strains were precultured in the seed medium CGIII at $30^{\circ} \mathrm{C}$ and 200 r min ${ }^{-1}$ until the $A_{600}$ reached $12.1 \mathrm{~mL}$ of seed culture was inoculated in a 500-mL baffled shake flask with $30 \mathrm{~mL}$ CGX medium [24]. The cells were grown in triplicate at $30^{\circ} \mathrm{C}$ and shaken at $120 \mathrm{r} \mathrm{min}^{-1}$. The $\mathrm{pH}$ was maintained at 7.0-7.2 by supplementation with ammonia.

Fed-batch fermentation was carried out in a 7.5-L bioreactor (BioFlo ${ }^{\circledR} /$ CelliGen ${ }^{\circledR} 115$, New Brunswick, USA) with a working volume of $2 \mathrm{~L} \mathrm{CGX} \mathrm{medium} \mathrm{containing} 20 \mathrm{~g} \mathrm{~L}^{-1}$ initial glucose. After $4 \mathrm{~h}$ of growth, from an $A_{600}$ of 0.2 to an
$A_{600}$ of 2.0, $0.8 \mathrm{mmol} \mathrm{L}^{-1}$ IPTG was added for induction of $\mathrm{P}_{t a c}$. The feed started after the residual sugar concentration was $<10 \mathrm{~g} \mathrm{~L}^{-1}$ with feed medium containing $400 \mathrm{~g} \mathrm{~L}^{-1}$ glucose. Temperature was maintained at $30^{\circ} \mathrm{C}$ using cooling water circulation. The $\mathrm{pH}$ was maintained at 7.2 by automated addition of ammonia and $100 \mathrm{mmol} \mathrm{L}^{-1} \mathrm{H}_{3} \mathrm{PO}_{4}$. Dissolved oxygen was determined using a $\mathrm{pO}_{2}$ electrode and maintained above $30 \%$ saturation level by variation of the stirrer speed.

\subsection{Determination of intracellular metabolites-sam- pling, quenching, and extraction}

Bioreactor-grown cells were harvested at mid-exponential growth phase. $5 \mathrm{~mL}$ of culture was injected into the quenching solutions $\left(60 \%\right.$ aqueous methanol $+70 \mathrm{mmol} \mathrm{L}^{-1}$ Hepes) and directly centrifuged at $5200 \times g$ at $-20^{\circ} \mathrm{C}$. After the removal of the supernatant, cell pellets were immediately frozen at $-80^{\circ} \mathrm{C}[28,29]$. Subsequently, cell pellets were extracted three times by boiling at $90^{\circ} \mathrm{C}$ for $5 \mathrm{~min}$ as described previously [30]. The obtained extracts were evaporated to dryness in a pump under vacuum at room temperature. After suspension of the extracts in $500 \mu \mathrm{L}$ of water, cell debris was removed by centrifugation for $10 \mathrm{~min}$ at $5500 \times g$. After decanting, the supernatant was stored at $-80^{\circ} \mathrm{C}$ until further analysis [29]. To reduce variations in feeding and maintain accuracy, SER- 0 and SER- 8 were grown in CGX minimal medium with $40 \mathrm{~g} \mathrm{~L}^{-1}$ glucose in a batch-fermentation process. 


\subsection{Analytical methods}

The glucose concentration was measured using an SBA40D biosensor analyzer (Institute of Biology of Shandong Province Academy of Sciences, Shandong, China). Cell dry mass (CDM) was estimated with a spectrophotometer (1.0 $A_{600}$ is equivalent to $\left.0.27 \mathrm{~g} \mathrm{~L}^{-1} \mathrm{CDM}\right)$. Amino acids in the culture supernatant were determined using high performance liquid chromatography with a Zorbax Eclipse $\mathrm{XDB}-\mathrm{C}_{18}$ column $(4.6 \mathrm{~mm} \times 250 \mathrm{~mm}, 5 \mu \mathrm{m}$; Agilent $)$ at $40^{\circ} \mathrm{C}$ after derivatization with 2,4-dinitrofluorobenzene. Mobile phase A was $55 \%(\mathrm{v} / \mathrm{v})$ acetonitrile whereas mobile phase D consisted of $40 \mathrm{mmol} \mathrm{L}{ }^{-1} \mathrm{KH}_{2} \mathrm{PO}_{4}$ at $\mathrm{pH}$ 7.0-7.2. The flow rate of the mobile phase was $1 \mathrm{~mL} \mathrm{~min}^{-1}$.

\subsection{Elementary mode and metabolic flux analysis}

The $C$. glutamicum metabolic network was constructed as shown in Figure 3. The model was based on utilizing glucose as the carbon source. The complete set of reactions used for the elementary mode analysis is listed in Appendix Table 2 in the electronic version. The pathway details were collected from the KEGG database (http://www.kegg.com), comprising reactions involved in the glycolysis pathway (EMP), the pentose phosphate pathway (PPP), the tricarboxylic acid cycle (TCA), anaplerotic reaction converting phosphoenol pyruvate (PEP) into oxaloacetic acid (OAA) and synthetic reactions for serine, glycine, pyruvate, alanine, valine, isoleucine, leucine, glutamine, lysine, succinic acid and histidine. Intracellular fluxes were estimated from measurements of metabolite uptake and output rates (exchange fluxes) by a pseudo-steady-state approximation $[31,32]$. Elementary mode analysis was constructed using MATLAB software version 7.0 (MathWorks). Stoichiometric coefficients of the external metabolites present in the modes were written in the form of a matrix equation (eq. (1)):

$$
A x(t)=r(t),
$$

where $A$ is an $m \times n$ matrix of stoichiometric coefficients, the number of rows was equal to the number of external metabolites and the number of columns was equal to the number of modes [32].

The stoichiometric model in this study (Appendix Tables 2 and 3 in the electronic version) consists of 29 reactions and 17 compounds; the degree of freedom (DF) is $29-17=12$, which means that changes in the rate of twelve compounds should be measured to determine all of the reaction rates in the metabolic network. External metabolites considered to generate flux distribution maps during the course of the fermentation were measured for quantification, including glucose, serine, glycine, alanine, valine, isoleucine, leucine, glutamine, lysine, succinic acid, histidine and biomass.

\section{Results}

\subsection{Metabolic engineering of $C$. glutamicum for serine production}

Our overall strategy to optimize C. glutamicum for L-serine production was to simultaneously enhance its biosynthesis and reduce its degradation (Figure 1; also see introduction section). For the latter, an $s d a A$-null strain, SER-1, was first created to block the degradation of L-serine to yield pyruvate. The $g l y R$ gene was deleted subsequently in SER-1 to create the $s d a A-$ and $g l y R$-double-null mutant, SER-2. SER-2 lacks the transcriptional activator GlyR to up-regulate the SHMT expression in the stationary phase and is supposed to reduce the conversion of L-serine to glycine. In order to enhance the L-serine biosynthesis, strain SER-3 was created from SER-2 to have the endogenous PGDH replaced with a C-terminal 197 amino acid truncated one. Based on SER-3, three further steps were applied to increase the supply of the key precursor substrate for L-serine biosynthesis, 3-phosphoglycerate, by over-expressing either PGK (SER-5) or the feedback-resistant PGDH variant $\left(\mathrm{PGDH}^{\mathrm{r}}\right)$ (SER-6). Following this strategy, a series of mutant strains were generated to facilitate the metabolic dissection of the contribution of these individual enzymes to L-serine accumulation. This design also made the analysis of the effects of these gene modifications on the metabolism of C. glutamicum possible (Figure 1).

\subsection{Metabolic analysis of the mutant strains for L-serine production}

Use of both the SER-1 and SER-2 strains did not improve the L-serine production, indicating that blocking the degradation of L-serine to pyruvate and reducing the conversion of L-serine to glycine hardly contributed to L-serine accumulation. Strain SER-3 can accumulate L-serine at a level of $0.01 \mathrm{mmol} \mathrm{L}^{-1}$, suggesting that the rate-limiting enzyme, $\mathrm{PGDH}$, might be a minor metabolic node for L-serine accumulation in C. glutamicum and further improvement of the metabolic flux toward L-serine biosynthesis was necessary. Strain SER-3 was engineered to harbor plasmid-based $p g k$ (SER-5) or feedback-resistant $\operatorname{serA}^{\mathrm{r}}$ (SER-6). Use of these two strains further increased L-serine accumulation, suggesting that increasing the supply of precursors contributed to L-serine biosynthesis. Then, $\operatorname{pgk}$ and $\operatorname{ser}^{\mathrm{r}}$ were co-expressed in strain SER-3. The resulting strain, SER-8, showed a significant increase in L-serine accumulation, reaching a maximal level of $(0.425 \pm 0.019) \mathrm{mmol} \mathrm{L}^{-1}$ during early fermentation after $9 \mathrm{~h}$ of cultivation, a 14.16-fold increase over the control strain SER-4 in shake flasks.

\subsection{Physiological evaluation of SER-8 on an increased scale}

\subsubsection{The fermentation of SER-8 in a 7.5-L bioreactor}

In order to assess the overall consequences of these five 
genetic modifications on strain growth and L-serine production, a fed-batch fermentation in glucose minimal medium was performed in a 7.5-L bioreactor. As shown in Figure 2A, the fermentation process exhibited five distinct phases: Phase I, pure growth (0-15 h); Phase II, transition from pure growth to L-serine production (15-22 h); Phase III, L-serine accumulation at the expense of growth (22-28 h); Phase IV, L-serine productivity loss and a small degree of restoration of growth (28-37 h); and Phase V, death and L-serine yield stability (37-60 h). The specific growth of strain SER-8 was $0.26 \mathrm{~h}^{-1}$, significantly lower than that of the control strain, SER-0 $\left(\mu_{\max }=0.49 \mathrm{~h}^{-1}\right)$. The maximal CDM reached $16.71 \mathrm{~g}$
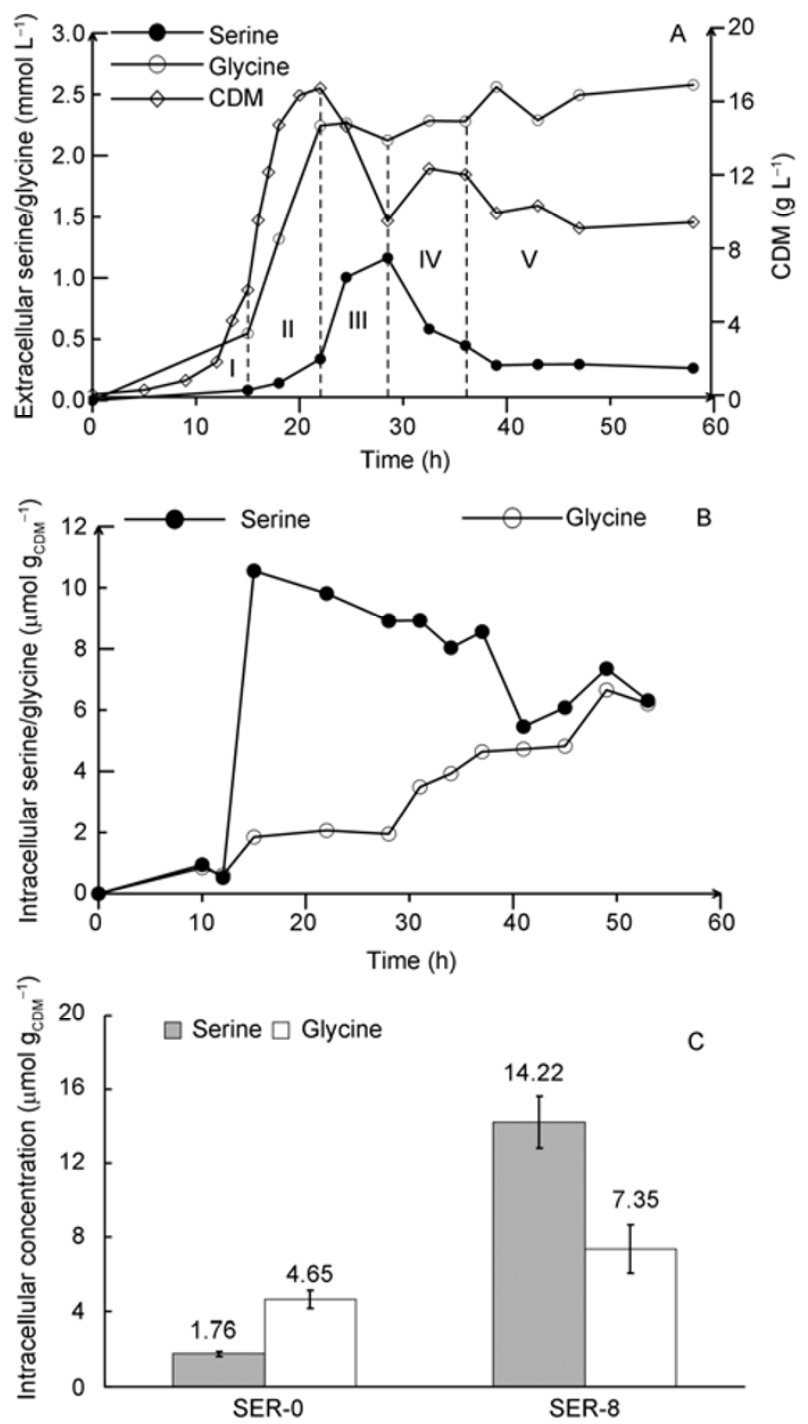

Figure 2 Physiological variation of SER-8 in a 7.5-L bioreactor. A, Performance of strain SER-8 in a fed-batch fermentation on CGX minimal medium with $20 \mathrm{~g} \mathrm{~L}^{-1}$ glucose showing growth, the accumulation of extracellular L-serine and glycine. B, Variations of intracellular L-serine and glycine levels depending on the incubation period in the medium. C, Intracellular pools of L-serine and glycine in mid-exponential phase; to reduce variation of feeding glucose, SER-0 and SER-8 were grown in CGX minimal medium containing $40 \mathrm{~g} \mathrm{~L}^{-1}$ glucose in a batch-fermentation. Error bars show the standard deviation for triple biological samples.
$\mathrm{L}^{-1}$ after $22 \mathrm{~h}$ of cultivation and the maximal L-serine production reached $1.21 \mathrm{mmol} \mathrm{L}^{-1}$ after $28 \mathrm{~h}$.

\subsubsection{The extra- and intra-cellular variation of L-serine and glycine}

Extracellular L-serine reached a maximum at $28 \mathrm{~h}$ and then decreased until the end of the fermentation. The extracellular glycine accumulated at the beginning of cultivation and reached as high as $2.24 \mathrm{mmol} \mathrm{L}^{-1}$. After that, the glycine level kept increasing slightly along with the utilization of L-serine even after completion of Phase IV. For all fermentative phases, the extracellular L-serine level was much lower than glycine (Figure 2A). On the other hand, the intracellular L-serine level reached a maximum of $10.56 \mu \mathrm{mol}$ $\mathrm{g}_{\mathrm{CDM}}{ }^{-1}$ after about $15 \mathrm{~h}$ of cultivation prior to its excretion (Figure 2B) and declined afterwards. On the contrary, the intracellular glycine was significant lower than the intracellular L-serine and increased continuously during the whole fermentation process (Figure 2B).

\subsubsection{The intracellular pool of L-serine and glycine}

A comparison of the intracellular pool of L-serine and glycine from SER-8 to that of SER-0 indicated that an 8.08 -fold increase was achieved, i.e., from $(1.76 \pm 0.11)$ to $(14.22 \pm 1.41) \mu \mathrm{mol} \mathrm{g}_{\mathrm{CDM}}{ }^{-1}$ while the glycine pool increased by $34 \%$, from $(4.65 \pm 0.47)$ to $(7.35 \pm 1.35) \mu \mathrm{mol} \mathrm{g}_{\mathrm{CDM}}{ }^{-1}$ (Figure 2C). The increased intracellular pool of both L-serine and glycine in SER-8 indicated that a higher flux was indeed directed into the L-serine biosynthesis pathway in the desired manner as designed. Interestingly, it was also noticed that the intracellular L-serine pool was much lower than glycine in SER-0 (Figure 2C). In contrast, SER-8 showed a higher intracellular pool of L-serine than glycine.

\subsection{Comparative metabolic flux analysis of strains SER-0 and SER-8}

To compare the flux distribution of the glycolysis pathway (EMP), the pentose phosphate pathway (PPP), the TCA cycle and the L-serine metabolism pathway in SER-0 and SER-8, the relative fluxes of the metabolic pathway were normalized to the glucose uptake rate $(\%)$ as shown in Figure 3. Significant changes in the flux distribution of EMP and PPP were observed between the SER-0 and SER-8 strains. The ratio of EMP flux in strain SER-8 significantly increased, implying the over-expression of PGK redirected more flux from PPP toward EMP. As a result of the increased EMP, SER- 8 exhibited an almost $27 \%$ increase of the relative flux through the TCA cycle. Despite the fact that the supply of 3-phosphoglycerate increased, only a relatively small flux $(0.68 \%)$ was assigned to the L-serine biosynthesis pathway. The flux toward L-serine biosynthesis was elevated to 2 fold, indicating the over-expression of $\mathrm{PGDH}^{\mathrm{r}}$ redirected more flux to the L-serine biosynthesis pathway. In addition, the flux from L-serine to glycine 


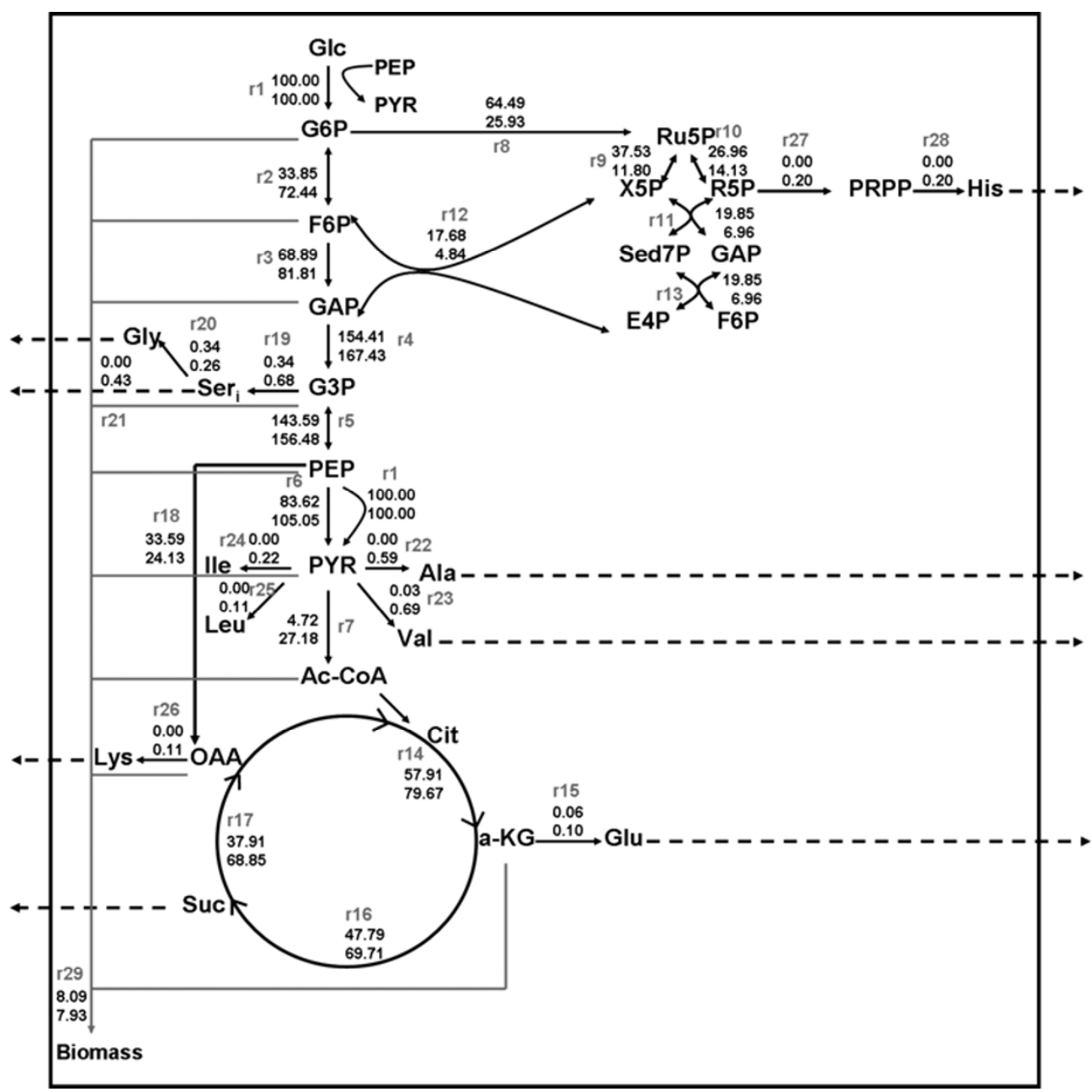

Figure 3 In vivo carbon flux distribution in the central metabolism of the control strain SER-0 (upper numbers of each pair) and the mutant SER-8 (lower numbers of each pair) during growth on glucose. All fluxes are expressed as the molar percentage of the mean specific glucose uptake rate $\left(2.47 \mathrm{mmol}^{-1}\right.$ $\mathrm{h}^{-1}$ for the control strain SER-0, $1.58 \mathrm{mmol} \mathrm{g}^{-1} \mathrm{~h}^{-1}$ for the mutant SER-8), which is defined as $100 \%$. A replicate batch experiment showed consistent results with a standard deviation of $10 \%$.

dropped by $24 \%$, suggesting the conversion of L-serine to glycine was reduced by deleting the activator GlyR to down-regulate SHMT at the transcriptional level. Furthermore, the extracellular L-serine shunt flux increased to $0.43 \%$ in strain SER-8. In contrast, the total L-serine in strain SER-0 was utilized for the generation of glycine, resulting in no net L-serine outside the cell for SER-0.

\section{Discussion}

In this study, a systematical investigation was performed on C. glutamicum to determine the effects of different genetic modifications on L-serine production. The mutant SER-8, a combination of five modified genes in C. glutamicum, exhibited a higher L-serine production (Figure 2A). According to the changes in flux distribution between strain SER-8 and SER-0 (control strain), over-expressing PGK can "push" carbon flux into the G3P pool, whereas engineering of
$\mathrm{PGDH}^{\mathrm{r}}$ can "pull" the carbon flux from the G3P pool into the L-serine biosynthesis pathway. This demonstrates that increases of the supply precursors are responsible for improving the flux distribution in L-serine biosynthesis.

The conversion of L-serine to glycine is the main degradation pathway of L-serine in C. glutamicum. It is impossible to disrupt the glyA gene since this reaction provides the majority of the $C_{1}$ units for cell growth [12]. When the expression of the glyA gene was controlled under the $\mathrm{P}_{t a c}$ promoter, L-serine accumulation was obviously increased in the absence of IPTG and simultaneously the growth of cells was seriously reduced [20]. Nevertheless, strong inhibition of glyA expression resulted in apparent instability of the engineered strain [16]. Our results showed that despite the deletion of the activator GlyR was believed to be responsible for decreasing the conversion of L-serine to glycine to a certain degree, the ratio of flux toward glycine was still $38.2 \%$. It further demonstrated that the conversion of L-serine to glycine is a rate-limiting step for L-serine accu- 
mulation. Therefore, controlling the glyA expression under a promoter of relatively weak activity would further reduce the conversion of L-serine to glycine.

L-Serine synthesis and accumulation was probably induced by growth limitation in these strains. This notion was supported by the observation of the decreases in the specific growth rate and biomass yield. The genetic modifications in C. glutamicum had a significant impact on the maximum growth rate $\left(\mu_{\max }\right)$ in accordance with previous reports [20]. Amino acid production under conditions of general growth limitations has been described elsewhere [18,33]. The decrease of the specific growth rate reflected a redirection of carbon flux due to genetic modifications of the L-serine biosynthetic pathway toward anabolic routes, and the co-metabolism of L-serine with glucose has shown that a distinct metabolic switch occurs-from the stage of incorporation of L-serine into biomass [9]. The growth maintenance during the stationary phase partially occurred at the expense of L-serine, which was evident from the gradual decrease in the concentration of L-serine and a concomitant sharp increase of glycine (Figures 2A and B). The positive effect of the genetic modifications on L-serine formation was diminished by simultaneous reutilization of L-serine to sustain physiological fitness. This suggests that L-serine biosynthesis occurred mainly at the expense of growth, and it would be optimal to decrease the specific growth rate for L-serine accumulation. Taken together, our findings imply that L-serine pool management was well correlated with the viability of $C$. glutamicum.

Interestingly, extracellular glycine continuously accumulated during all fermentative phases. For E. coli, the glycine cleavage (GCV) system converts glycine to 5,10-methylenetetrahydrofolate [34]. This system plays an important role in maintaining the intracellular homeostatic balance between glycine and concentrations of $C_{1}$ units [34]. However, glycine is an end-product without an efficient supply as a direct precursor in C. glutamicum [35]. Therefore, the export of redundant glycine might be an alternative way for $C$. glutamicum to maintain an intracellular glycine metabolic equilibrium. These findings make it possible to consume the intracellular redundant glycine by integrating the GCV system of E. coli into the $C$. glutamicum chromosome. In this way, the GCV system will generate more active $\mathrm{C}_{1}$ units to provide a balance between cell growth and L-serine accumulation in $C$. glutamicum. Therefore, improving the generation of $\mathrm{C}_{1}$ units by introducing the GCV system to degrade the excessive glycine is a promising target for the metabolic engineering of $C$. glutamicum for L-serine production.

In this study, several systems metabolic engineering strategies have been attempted to develop a L-serine production strain. Since the entire metabolic and regulatory networks are woven into a global system in an integrated manner, some target gene engineering is still necessary until an optimal strategy is obtained [36,37]. The work reported herein provides a framework of information on the direct fermentative production of L-serine from glucose.

We are grateful to Fan ZhenChuan, Deng AiHua and Zhang GuoQiang for the critical evaluation of the manuscript. This work was supported by grants from Ministry of Science and Technology of China (Grant Nos. 2008ZX09401-05 and 2010ZX09401-403), the National Natural Science Foundation of China (Grant No. 31100074), and Chinese Academy of Sciences (Grant No. XBXA-2011-009).

1 Furuya S. An essential role for de novo biosynthesis of L-serine in CNS development. Asia Pac Clin Nutr, 2008, 17: 312-315

2 Furuya S, Makino A, Hirabayashi Y. An improved method for culturing cerebellar Purkinje cells with differentiated dendrites under a mixed monolayer setting. Brain Res Protoc, 1998, 3: 192-198

3 Pizer L I, Potochny M L. Nutritional and regulatory aspects of serine metabolism in Escherichia coli. J Bacteriol, 1964, 88: 611-619

4 McNeil J B, Bognar A L, Pearlman R E. In vivo analysis of folate coenzymes and their compartmentation in Saccharomyces cerevisiae. Genetics, 1996, 142: 371-381

5 Gelling C L, Piper M D W, Hong S P, et al. Identification of a novel one-carbon metabolism regulon in Saccharomyces cerevisiae. J Biol Chem, 2004, 279: 7072-7081

6 Stauffer G V. Biosynthesis of serine, glycine, and one-carbon units. In: Neidhardt F C Curtiss III R, Ingraham J L, Lin E C C, et al., eds. Escherichia coli and Salmonella: Cellular and Molecular Biology. 2nd ed. Washington D.C.: ASM Press, 1996. 506-513

7 Marx A, deGraaf A A, Wiechert W, et al. Determination of the fluxes in the central metabolism of Corynebacterium glutamicum by nuclear magnetic resonance spectroscopy combined with metabolite balancing. Biotechnol Bioeng, 1996, 49: 111-129

8 Peters-Wendisch P, Netzer R, Eggeling L, et al. 3-Phosphoglycerate dehydrogenase from Corynebacterium glutamicum: the C-terminal domain is not essential for activity but is required for inhibition by L-serine. Appl Microbiol Biotechnol, 2002, 60: 437-441

9 Netzer R, Peters-Wendisch P, Eggeling L, et al. Cometabolism of a nongrowth substrate: L-serine utilization by Corynebacterium glutamicum. Appl Environ Microbiol, 2004, 70: 7148-7155

10 Haitani Y, Awano N, Yamazaki M, et al. Functional analysis of L-serine $O$-acetyltransferase from Corynebacterium glutamicum. FEMS Microbiol Lett, 2006, 255: 156-163

11 Ikeda M. Towards bacterial strains overproducing L-tryptophan and other aromatics by metabolic engineering. Appl Microbiol Biotechnol, 2006, 69: 615-626

12 Simic P, Willuhn J, Sahm H, et al. Identification of glyA (encoding serine hydroxymethyltransferase) and its use together with the exporter ThrE to increase L-threonine accumulation by Corynebacterium glutamicum. Appl Environ Microbiol, 2002, 68: 3321-3327

13 Schweitzer J E, Stolz M, Diesveld R, et al. The serine hydroxymethyltransferase gene glyA in Corynebacterium glutamicum is controlled by GlyR. J Biotechnol, 2009, 139: 214-221

14 Kubota K, Yokozeki K. Production of L-serine from glycine by Corynebacterium glycinophilum and properties of serine hydroxymethyltransferase, a key enzyme in L-serine production. J Ferment Bioeng, 1989, 67: 387-390

15 Izumi Y, Yoshida T, Miyazaki S S, et al. L-Serine production by a methylotroph and its related enzymes. Appl Microbiol Biotechnol, 1993, 39: 427-432

16 Stolz M, Peters-Wendisch P, Etterich H, et al. Reduced folate supply as a key to enhanced L-serine production by Corynebacterium glutamicum. Appl Environ Microbiol, 2007, 73: 750-755

17 Becker J, Zelder O, Häfner S, et al. From zero to hero-design-based systems metabolic engineering of Corynebacterium glutamicum for L-lysine production. Metab Eng, 2011, 13: 159-168

18 Holátko J, Elišáková V, Prouza M, et al. Metabolic engineering of the L-valine biosynthesis pathway in Corynebacterium glutamicum 
using promoter activity modulation. J Biotechnol, 2009, 139: 203 210

19 Park S D, Lee J Y, Sim S Y, et al. Characteristics of methionine production by an engineered Corynebacterium glutamicum strain. Metab Eng, 2007, 9: 327-336

20 Peters-Wendisch P, Stolz M, Etterich H, et al. Metabolic engineering of Corynebacterium glutamicum for L-serine production. Appl Environ Microbiol, 2005, 71: 7139-7144

21 Sambrook J, Fritsch E F, Maniatis T. Molecular Cloning: A Laboratory Manual. 2nd ed. New York: Cold Spring Harbor Laboratory Press, 1989

22 Schäfer A, Tauch A, Jäger W, et al. Small mobilizable multi-purpose cloning vectors derived from the Escherichia coli plasmids pK18 and pK19: selection of defined deletions in the chromosome of Corynebacterium glutamicum. Genetics, 1994, 145: 69-73

23 Jakoby M, Ngouoto-Nkili C E, Burkovski A. Construction and application of new Corynebacterium glutamicum vectors. Biotechnol Tech, 1999, 13: 437-441

24 Cremer J, Eggeling L, Sahm H. Control of the lysine biosynthesis sequence in Corynebacterium glutamicum as analyzed by overexpression of the individual corresponding genes. Appl Environ Microbiol, 1991, 57: 1746-1752

25 van der Rest M E, Lange C, Molenaar D. A heat shock following electroporation induces highly efficient transformation of Corynebacterium glutamicum with xenogeneic plasmid DNA. Appl Microbiol Biotechnol, 1999, 52: 541-545

26 Jäger W, Schäfer A, Pühler A, et al. Expression of the Bacillus subtilis $s a c B$ gene leads to sucrose sensitivity in the gram-positive bacterium Corynebacterium glutamicum but not in Streptomyces lividans. J Bacteriol, 1992, 174: 5462-5465

27 Zhang Y, Shang X, Deng A, et al. Genetic and biochemical characterization of Corynebacterium glutamicum ATP phosphoribosyltransferase and its three mutants resistant to feedback inhibition by histidine. Biochimie, 2012, 94: 829-838
28 Persicke M, Plassmeier J, Neuweger H, et al. Size exclusion chromatography - an improved method to harvest Corynebacterium glutamicum cells for the analysis of cytosolic metabolites. J Biotechnol, 2011, 155: 266-267

29 Taymaz-Nikerel H, de Mey M, Ras C, et al. Development and application of a differential method for reliable metabolome analysis in Escherichia coli. Anal Biochem, 2009, 386: 9-19

30 Klimacek M, Krahulec S, Sauer U, et al. Limitations in xylosefermenting Saccharomyces cerevisiae, made evident through comprehensive metabolite profiling and thermodynamic analysis. Appl Environ Microbiol, 2010, 76: 7566-7574

31 Krömer J O, Wittmann C, Schröder H, et al. Metabolic pathway analysis for rational design of L-methionine production by Escherichia coli and Corynebacterium glutamicum. Metab Eng, 2006, 8: 353-369

32 Vallino J J, Stephanopoulos G. Metabolic flux distributions in Corynebacterium glutamicum during growth and lysine overproduction. Biotechnol Bioeng, 1993, 41: 633-646

33 Radmacher E, Vaitsikova A, Burger U, et al. Linking central metabolism with increased pathway flux: L-valine accumulation by Corynebacterium glutamicum. Appl Environ Microbiol, 2002, 68: 22462250

34 Stauffer L T, Stauffer G V. Role for the leucine-responsive regulatory protein (Lrp) as a structural protein in regulating the Escherichia coli gcvTHP operon. Microbiology, 1999, 145: 569-576

35 Kalinowski J, Bathe B, Bartels D, et al. The complete Corynebacterium glutamicum ATCC 13032 genome sequence and its impact on the production of L-aspartate-derived amino acids and vitamins. J Biotechnol, 2003, 104: 5-25

36 Han M J, Lee S Y. The Escherichia coli proteome: past, present, and future prospects. Microbiol Mol Biol Rev, 2006, 70: 362-439

37 Kjeldsen K R, Nielsen J. In silico genome-scale reconstruction and validation of the Corynebacterium glutamicum metabolic network. Biotechnol Bioeng, 2009, 102: 583-597

Open Access This article is distributed under the terms of the Creative Commons Attribution License which permits any use, distribution, and reproduction in any medium, provided the original author(s) and source are credited. 